\title{
Thyroid Storm with Multiple Organ Failure, Disseminated Intravascular Coagulation, and Stroke with a Normal Serum FT3 Level
}

\author{
Yuko Harada $^{1}$, Hisanao Akiyama ${ }^{2}$, Tatsuji Yoshimoto ${ }^{1}$, Yasuko Urao ${ }^{3}$, \\ Munekazu Ryuzaki ${ }^{4}$ and Michiko Handa ${ }^{4}$
}

\begin{abstract}
Thyroid storm is a rare disorder with a sudden onset, rapid progression and high mortality. We experienced a case of thyroid storm which had a devastating course, including multiple organ failure (MOF), severe hypoglycemia, disseminated intravascular coagulation (DIC), and stroke. It was difficult to make a diagnosis of thyroid storm in the present patient, because she did not have a history of thyroid disease and her serum FT3 level was normal. Clinicians should be aware that thyroid storm can occur even when there is an almost normal level of thyroid hormones, and that intensive anticoagulation is required for patients with atrial fibrillation to prevent stroke after thyroid storm.
\end{abstract}

Key words: thyroid storm, cardiogenic embolism, Graves' disease, disseminated intravascular coagulation, multiple organ failure

(Intern Med 51: 2379-2383, 2012)

(DOI: 10.2169/internalmedicine.51.7974)

\section{Introduction}

Thyroid storm, or thyrotoxic crisis, is a hypermetabolic state induced by the excessive release of thyroid hormones in patients with thyrotoxicosis. It has been reported that the mortality rate is higher than $20 \%$ even with a rapid diagnosis and treatment (1). The diagnostic criteria used worldwide were established by Burch and Wartofsky in 1993 (2). The Japan Thyroid Association and Japan Endocrine Society established new criteria in 2012 (3). It is helpful to use both criteria to make a rapid diagnosis. We experienced a case of thyroid storm with devastating manifestations which was difficult to diagnose. Both criteria helped us to make a diagnosis and to save the patient's life.

\section{Case Report}

A 62-year-old woman was admitted to the hospital due to a weight loss of $13 \mathrm{~kg}$ over 3 months. She also had experienced a fever and productive cough for three days. On the day of admission she had dyspnea, palpitations and diarrhea. She was not taking any medication. Her past medical history included pneumonia, but the details were unknown.

An examination on admission revealed a thin woman of $156 \mathrm{~cm}$ in height and $40 \mathrm{~kg}$ in weight, with vital signs as follows: body temperature, $39.2^{\circ} \mathrm{C}$; heart rate, 152 beats/ min; and blood pressure, 100/72 mmHg. Her consciousness level was E4, V5, M6 and she scored a total of 15 points on the Glasgow Coma Scale. Her level of consciousness was I0 on the Japan Coma Scale. Her heartbeat was a galloping rhythm, and coarse crackles were heard in the right upper field of the lung. Exophthalmos, remarkable sweating, and finger tremors were observed, but there was no goiter.

The patient's CBC count showed increased white cells at $13,200 / \mu \mathrm{L}$, a low hemoglobin level of $10.6 \mathrm{~g} / \mathrm{dL}$, and a decreased platelet count of $146,000 / \mu \mathrm{L}$. The total cholesterol was decreased to $76 \mathrm{mg} / \mathrm{dL}$, the CRP was increased to 12.49

\footnotetext{
${ }^{1}$ Department of Cardiology, Kawasaki Municipal Ida Hospital, Japan, ${ }^{2}$ Department of Neurology, St. Marianna University School of Medicine, Japan, ${ }^{3}$ Department of Otorhinolaryngology, Nihon Koukan Hospital, Japan and ${ }^{4}$ Department of Internal Medicine, Kawasaki Municipal Ida Hospital, Japan

Received for publication April 16, 2012; Accepted for publication June 17, 2012

Correspondence to Dr. Yuko Harada, adayuko1219@yahoo.co.jp
} 

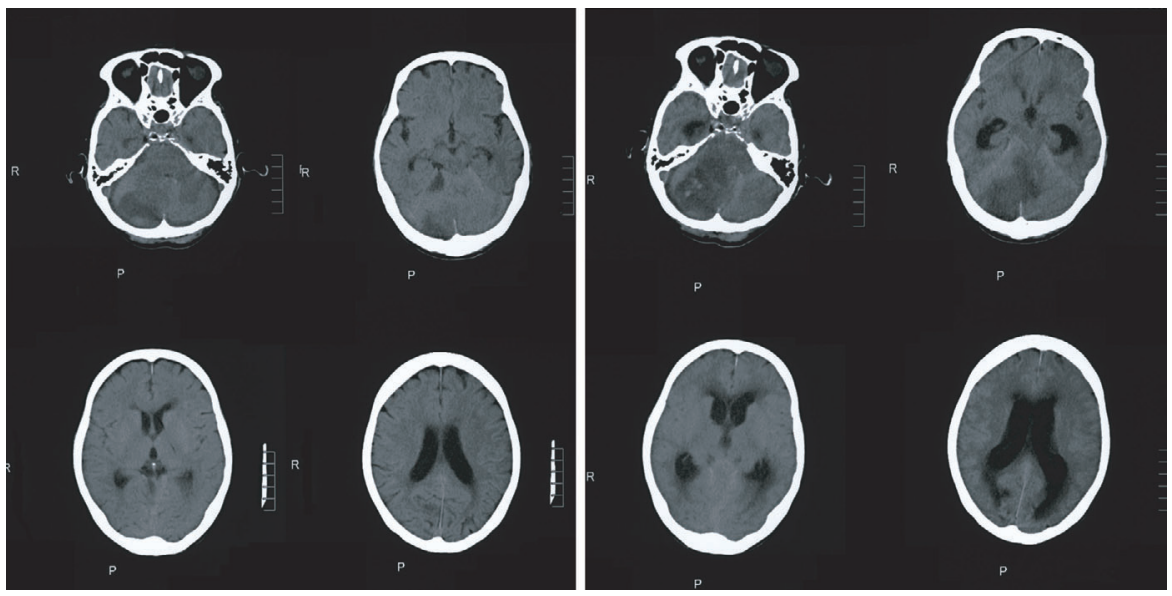

Figure 1. Left: Head CT scan on 24th day of admission. There is a localized low density area in the right hemisphere of cerebellum, which was diagnosed as right cerebellar infarction. Right: Head CT scan on the next day. Hemorrhagic infarction in the posterior fossa and hydrocephalus are shown. The brain stem is compressed by cerebellar edema.

$\mathrm{mg} / \mathrm{dL}$, and the BNP was increased to $593.2 \mathrm{pg} / \mathrm{mL}$. It was suggested that the patient had hyperthyroidism because she had a high FT4 level of $2.92 \mathrm{ng} / \mathrm{dL}$ (normal range 0.701.48), and an extremely low level of TSH, which was below $0.0100 \mu \mathrm{IU} / \mathrm{mL}$, but her FT3 was normal at $2.90 \mathrm{pg} / \mathrm{mL}$ (normal range 1.71-3.71). Her thyroid receptor antibody (TRAb) titer increased to $34.6 \%$, one week after hospital admission. An electrocardiogram showed atrial fibrillation with a rapid ventricular response. Chest X-rays showed cardiomegaly, pulmonary congestion and infiltration in the right upper field.

\section{Clinical course}

The primary diagnosis was acute heart failure of NYHA class IV and Killip class III with pneumonia and hyperthyroidism. Heparin at 10,000 IU/day was given to prevent the development of an embolism because of the atrial fibrillation sustained at the rapid heart rate. Ceftriaxone (CTRX, 2 $\mathrm{g}$ /day) was given for the treatment of pneumonia and furosemide (20 mg/day) was given for the heart failure. Verapamil at $120 \mathrm{mg} /$ day was also given for the sustained tachycardia. On the 2nd day of admission, the patient showed mental excitement and confusion. Her tachycardia worsened, so $30 \mathrm{mg} /$ day propranolol was given to control the heart rate. On the 3rd day of admission she suddenly lost consciousness with her eyes widely opened, and fell into a deep coma and shock. Dopamine and dobutamine were given for the treatment of shock. The laboratory data showed hyperkalemia of $6.9 \mathrm{mEq} / \mathrm{L}$ and remarkable hypoglycemia of $6 \mathrm{mg} /$ dL.

At that point, we diagnosed her with thyroid storm, and started to give her $20 \mathrm{mg}$ of thiamazole every 6 hours orally. Her hypoglycemia was corrected with an intravenous injection of dextrose solution. We also gave her $100 \mathrm{mg}$ hydrocortisone every 8 hours intravenously. On the next day, her platelet count decreased to $75,000 / \mu \mathrm{L}$, the antithrombin-III level was decreased to $27.0 \%$, her prothrombin time was less than $10.0 \%$, and the FDP-D dimer level was increased to 53.6, which was compatible with disseminated intravascular coagulation (DIC). Her BUN was increased to $76.6 \mathrm{mg} /$ $\mathrm{dL}$ and her creatinine was increased to $2.7 \mathrm{mg} / \mathrm{dL}$, which indicated acute renal failure. Her total bilirubin was also increased to $1.6 \mathrm{mg} / \mathrm{dL}$, AST was increased to $2,534 \mathrm{IU} / \mathrm{L}$, and ALT was increased to $497 \mathrm{IU} / \mathrm{L}$, which was suggestive of hepatic failure. Thereafter, she developed DIC and multiple organ failure (MOF), but both of these improved during the following two weeks by treatment with gabexate mesilate (FOY), heparin, thiamazole and hydrocortisone.

The psychological symptoms of this case were characteristic. On admission she was talkative, but her mental status was within the normal range. On the second day, her mental excitement seemed to increase in proportion to her heart rate. On the 3rd day when she was endlessly speaking, she suddenly lost consciousness with her eyes wide open. Then her heart rate decreased, and she went into shock, falling into a deep coma. At this moment her blood glucose was only $6 \mathrm{mg} / \mathrm{dL}$, so the disturbance of consciousness was thought to be due to or worsened by the hypoglycemia. However, after the hypoglycemia was corrected by the intravenous injection of dextrose solution, the disturbance of consciousness was sustained at E2V2 M4 of the Glasgow Coma Scale for more than 8 hours. It took about a week until her mental status returned normal. Her thyroid hormones and TSH levels normalized in 10 days, so it seemed that her mental status recovered in proportion to the recovery from the thyroid storm.

On the 9th day of admission, she had recovered from DIC after the use of heparin. Heparin was switched to warfarin ( $2 \mathrm{mg} /$ day) for preventing cardiogenic embolism. Six days later, her PT-TNR was elevated to 4.88 and we therefore decreased the dose of warfarin. After another 4 days, the PTINR decreased to 1.18 , but we did not increase the dose of warfarin because we were planning to perform a biopsy of the thyroid. We stopped giving her warfarin to prepare for 


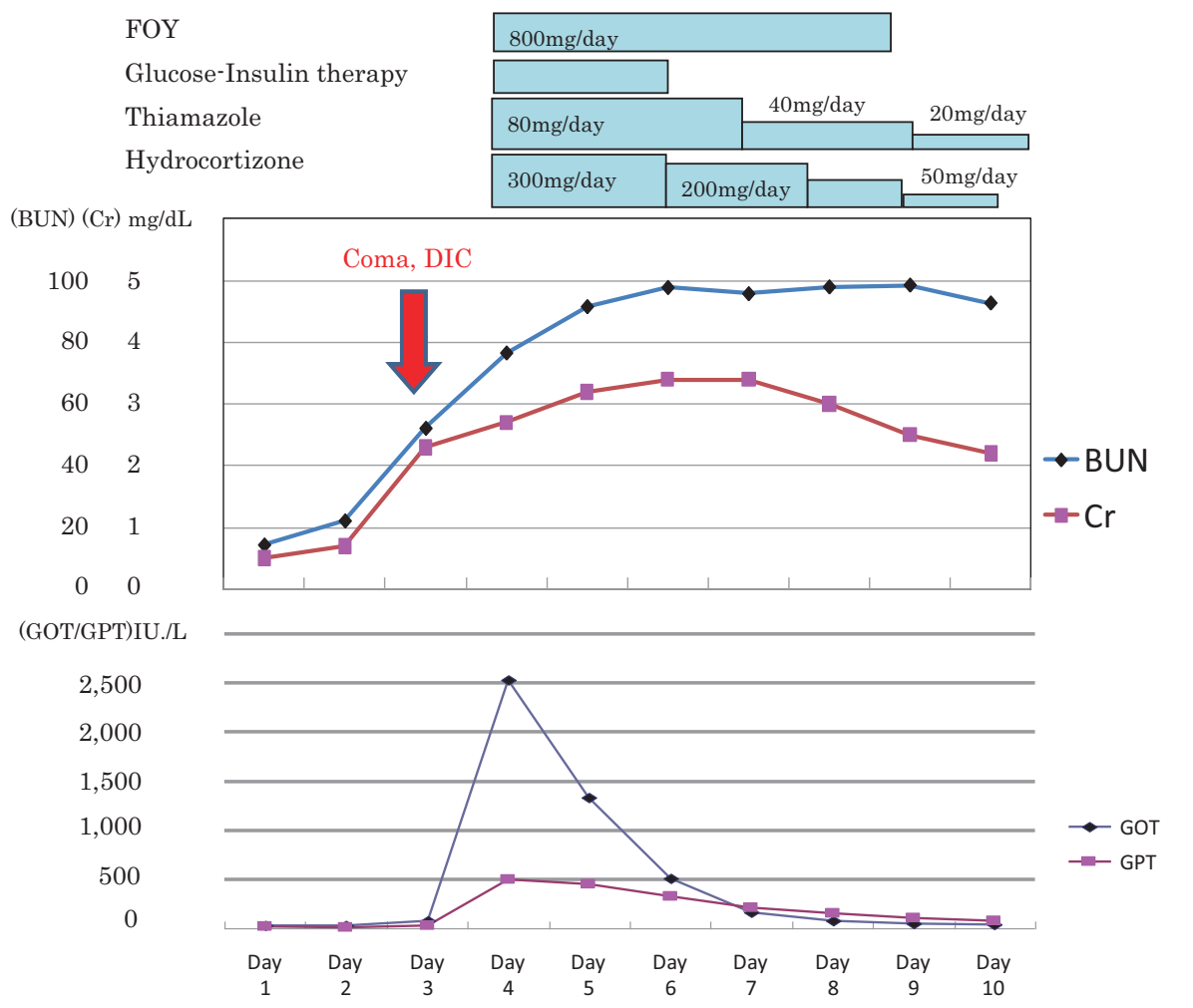

Figure 2. Clinical course and treatment. On the 3rd day of admission the patient fell into deep coma. Then she developed DIC and MOF, which were improved by treatment with FOY, heparin, thiamazole and hydrocortisone.

biopsy. Three days later, on the 24th day of admission, she suddenly developed a slight headache, nausea and dizziness. A head CT scan revealed a localized low density area in the right hemisphere of the cerebellum, which was diagnosed as a right cerebellar infarction (cardiogenic embolism) without bleeding (Fig. 1). Her PT-INR was 1.14. We gave her a free radical scavenger, edaravone, but she fell into deep coma the next day. Another head CT scan showed a hemorrhagic infarction in the posterior fossa and hydrocephalus. This obstructive hydrocephalus was remarkable, and the brain stem was compressed by cerebellar edema, so she was treated with decompressive surgery. The surgery was successful, and she was discharged from the hospital after rehabilitation. The patient currently visits our outpatient clinic every month, and is being treated with warfarin and thiamazole.

\section{Discussion}

A rapid diagnosis and proper treatment is the key to save the patients presenting with thyroid storm. This case was not recognized as thyroid storm on admission, because it was difficult to understand the condition of this patient. On admission, she was diagnosed with acute heart failure with hyperthyroidism and pneumonia, and appropriate treatment was started. But on the 3rd day, we could not immediately understand why she fell into a coma and developed hyperkalemia and hypoglycemia. The patient's progressive mental confusion and disturbance of consciousness were suggestive of thyroid storm, but her FT3 level was normal on admission. The hyperkalemia and hypoglycemia could have been caused by relative adrenal failure, which is known to accompany thyroid storm.

The criteria for thyroid storm used worldwide were established by Burch and Wartofsky (2). The points scored for this patient were 20 for the fever of $39.2^{\circ} \mathrm{C}, 20$ for delirium, 10 for diarrhea and nausea, 25 for the tachycardia of 152 beats/min, 15 for pulmonary edema, 10 for atrial fibrillation, and 10 for the precipitating event. Therefore, she scored a total of 110 out of 140 points, which was highly suggestive of thyroid storm. However, her thyroid hormone levels were not very high, and her FT3 level was normal. The patient did not have any history of thyroid disease. Ultrasonography showed a slightly enlarged thyroid with a poor blood flow, which is not a typical finding of Basedow's disease.

In 2008, the Japan Thyroid Association and Japan Endocrine Society established their diagnostic criteria for thyroid storm (4). According to these criteria, elevation of FT3 and/ or FT4 is essential. This patient had all five symptoms specified by the criteria, and since she met the criteria for both groups, we initially diagnosed her with thyroid storm.

This patient was admitted to our hospital in June, 2010 when the criteria for thyroid storm were being published by the Japan Thyroid Association and Japan Endocrine Society. If we had made the diagnosis on admission, we would have started treatment earlier, and the clinical course might not have been as devastating. 
There have been some other cases of thyroid storm with a normal serum FT4 level (2) or normal serum FT3 level (5-8) reported in the past. A case of thyroid storm with a normal level of both FT4 and FT3 was reported by Kuo et al. (9). The Japan Thyroid Association and Japan Endocrine Society diagnostic criteria clearly state that the elevation of FT3 and/or FT4 (3) is essential for a diagnosis, on the grounds that thyroid storm is not always associated with a remarkably high level of thyroid hormone (10). In a 5-years survey in Japan, several patients had normal serum T3 levels despite elevated serum T4 and low serum TSH levels (3), just like in our case. It has been suggested that the severity of thyroid storm does not necessarily correlate with the thyroid hormone levels $(2,11)$. Kuo et al. reported that the mechanism underlying the normalization of the thyroid hormone levels in thyroid storm may be similar to that of nonthyroidal illness syndrome, in which the induction of type 3 deiodinase and a reduction of type 1 deiodinase may contribute to the low T3 levels $(9,12)$.

The present case developed both MOF and DIC. Some previous cases of thyroid storm with MOF and/or DIC have been reported (13-17). Congestive heart failure, gastrointestinal dysfunction, and hepatic dysfunction are also often seen in thyroid storm, thus they are included in the diagnostic criteria proposed by Burch and Wartofskyb (2). The DIC might be caused by sepsis, which is a common trigger of thyroid storm. In our case, pneumonia was the trigger of the thyroid storm and DIC. The acute renal failure in this case was probably caused by dehydration, because the fractional excretion of filtrated $\mathrm{Na}$ (FENa) was less than $1 \%$. The patient's fever and use of diuretics could have caused dehydration, so care must be taken when using diuretics in patients with heart failure with hyperthyroidism.

We also considered this patient to have had a relative adrenal insufficiency. Shock, hypoglycemia, and hyperkalemia are known to be symptoms of adrenal failure. The blood test showed the patient's serum cortisol concentration to be 34.4 $\mu \mathrm{g} / \mathrm{dL}$ (normal range is 4.0-18.3), while the serum C-peptide concentration was $0.37 \mathrm{ng} / \mathrm{mL}$ (normal range, 0.61-2.09), and serum insulin concentration was $0.45 \mu \mathrm{U} / \mathrm{mL}$ (normal range 1.84-12.2). We therefore speculated that the critical status of this patient with shock, MOF, and DIC may have required more cortisol. However, even a slightly elevated level of cortisol might not have met her treatment needs.

The patient had a cerebellar stroke three weeks after the onset of thyroid storm. All the laboratory data had almost normalized, and her general condition was favorable. The stroke was probably caused by the poor INR control of warfarin. Her $\mathrm{CHADS}_{2}$ score was only 1 point, so she was not positive for anticoagulation. Even though we started with a small amount of warfarin, the PT-INR increased to 4.88 in 6 days. We therefore stopped the anticoagulation and planned a biopsy of the thyroid. She had a stroke, and her PT-INR was found to be only 1.14. Bar-Sela et al. reported the incidence of atrial fibrillation in 142 patients with thyrotoxicosis to be $21 \%$, while that of thromboembolic disease was $8.5 \%$, and that cerebral emboli accounted for $53 \%$ of the episodes (18). This incidence is not very high, but this report shows that patients with thyrotoxicosis should be carefully monitored for thromboemboli. Our case suggests that intensive control with warfarin is required for the patients with atrial fibrillation and thyrotoxicosis to prevent stroke, regardless of their $\mathrm{CHADS}_{2}$ score, especially after thyroid storm.

We experienced a case of thyroid storm with a normal serum FT3 level and a slightly elevated level of FT4 who developed MOF, DIC, and stroke. We were successfully able to save her life, but if we had made a diagnosis on admission and started the appropriate treatment earlier, she would not have developed such critical complications. Therefore, clinicians should be aware of the possibility of thyroid storm when a patient presents with heart failure with the symptoms of thyrotoxicosis, even if the thyroid hormone level is not highly elevated.

The authors state that they have no Conflict of Interest (COI).

\section{References}

1. Tietgens ST, Leinung MC. Thyroid storm. Med Clin North Am 79: 169-184, 1995.

2. Burch HB, Wartofsky L. Life-threatening thyrotoxicosis. Thyroid storm. Endocrinol Metab Clin North Am 22: 263-277, 1993.

3. Akamizu T, Satoh T, Isozaki O, et al. Diagnostic criteria and clinico-epidemiological features of thyroid storm based on a nationwide survey. Thyroid 2012 [Epub ahead of print], DOI: 10.1089/thy.2011-0334

4. The Guideline Committee for Thyroid Storm of Japan Thyroid Association and Japan Endocrine Society. Diagnostic Criteria for Thyroid Storm. 1st ed. [http://thyroid.umin.ac.jp/rinsho/crise1.pdf] 2008 (in Japanese).

5. Ogbuawa O, Johnson R. T4-thyroid storm in pernicious anemia. Arch Intern Med 140: 1245-1246, 1980.

6. Ahmad N, Cohen MP. Thyroid storm with normal serum triiodothyronine level during diabetic ketoacidosis. JAMA 245: 25162517, 1981.

7. Shimura H, Takazawa K, Endo T, Tawata M, Onaya T. T4-thyroid storm after CT-scan with iodinated contrast medium. J Endocrinol Invest 13: 73-76, 1990.

8. Kaplan MM. Clinical and laboratory assessment of thyroid abnormalities. Med Clin North Am 69: 863-880, 1985.

9. Kuo CS, Ma WY, Lin YC, Lin HD. Hepatic failure resulting from thyroid storm with normal serum thyroxine and triiodothyronine concentrations. J Chinese Med Assoc 73: 44-46, 2010.

10. Akamizu T. Thyroid storm. J Jpn Soc Intern Med 99: 763-768, 2010.

11. Wartofsky L. Thyrotoxic storm. In: Werner and Ingbar's Thyroid. 9th ed. Braverman LE, Utiger RD, Eds. Lippincott Williams \& Wilkins, Philadelphia, 2005: 651-657.

12. Koenig RJ. Modeling the nonthyroidal illness syndrome. Curr Opin Endocrinol Diabetes Obesity 15: 466-469, 2008.

13. Martin D. Disseminated intravascular coagulation precipitated by thyroid storm. South Med J 102: 193-195, 2009.

14. Chong HW, See KC, Phua J. Thyroid storm with multiorgan failure. Thyroid 20: 333-336, 2010.

15. Kobayashi C, Sasaki H, Kosuge K, et al. Severe starvation hypoglycemia and congestive heart failure induced by thyroid crisis, with accidentally induced severe liver dysfunction and dissemi- 
nated intravascular coagulation. Intern Med 44: 234-239, 2005.

16. Jiang YZ, Hutchinson KA, Bartelloni P, Manthous CA. Thyroid storm presenting as multiple organ dysfunction syndrome. Chest 118: 877-879, 2000.

17. Kanbay M, Sengul A, Guvener N. Trauma induced thyroid storm complicated by multiple organ failure. Chin Med J 118: 963-965, 2005.

18. Bar-Sela S, Ehrenfeld M, Eliakim M. Arterial embolism in thyrotoxicosis with atrial fibrillation. Arch Intern Med 141: 1191-1192, 1981.

(C) 2012 The Japanese Society of Internal Medicine http://www.naika.or.jp/imonline/index.html 